Supplement of Atmos. Meas. Tech. Discuss., 8, 11853-11891, 2015

http://www.atmos-meas-tech-discuss.net/8/11853/2015/

doi:10.5194/amtd-8-11853-2015-supplement

(C) Author(s) 2015. CC Attribution 3.0 License.

(c) (1)

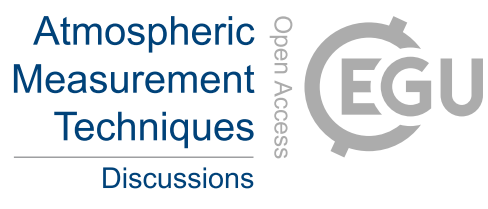

Supplement of

\title{
A sensitivity study on the retrieval of aerosol vertical profiles using the oxygen A-band
}

S. F. Colosimo et al.

Correspondence to: S. F. Colosimo (fedele@ atmos.ucla.edu)

The copyright of individual parts of the supplement might differ from the CC-BY 3.0 licence. 
In this Supplement, table charts for the complete set of resolutions are shown.

\begin{tabular}{cccccccc}
\hline \hline \multicolumn{2}{c}{ Resolution } & \multicolumn{6}{c}{$\boldsymbol{S}_{a}$ (relative error) } \\
\hline $\begin{array}{c}\Delta \nu \\
{\left[\mathrm{cm}^{-1}\right]}\end{array}$ & FWHM & 0.7 & 0.5 & 0.2 & 0.1 & 0.05 & 0.01 \\
{$\left[\mathrm{~cm}^{-1}\right]$} & $(83.7 \%)$ & $(70.7 \%)$ & $(44.7 \%)$ & $(31.6 \%)$ & $(22.4 \%)$ & $(10.0 \%)$ \\
\hline 1 & 5 & 1.94 & 1.82 & 1.51 & 1.29 & 1.12 & 0.78 \\
\hline 0.2 & 1 & 2.72 & 2.54 & 2.06 & 1.74 & 1.46 & 1.00 \\
\hline 0.1 & 0.5 & 3.22 & 3.05 & 2.60 & 2.26 & 1.92 & 1.25 \\
\hline 0.02 & 0.1 & 4.89 & 4.68 & 4.08 & 3.62 & 3.13 & 2.07 \\
\hline 0.01 & 0.05 & 5.94 & 5.68 & 4.97 & 4.44 & 3.89 & 2.59 \\
\hline \hline
\end{tabular}

Table S1. Complete set of resolutions DoF for different aerosol extinction profile uncertainties $\boldsymbol{S}_{a}$, as panel (a) in Fig.4. 


\begin{tabular}{cccccc}
\hline \hline \multicolumn{2}{c}{ Resolution } & \multicolumn{4}{c}{$\omega$ Urban scenario } \\
\hline $\begin{array}{c}\Delta \nu \\
{\left[\mathrm{cm}^{-1}\right]}\end{array}$ & $\begin{array}{c}\text { FWHM } \\
{\left[\mathrm{cm}^{-1}\right]}\end{array}$ & 0.8 & 0.85 & 0.9 & 0.95 \\
\hline 1 & 5 & 1.10 & 1.51 & 1.80 & 1.94 \\
\hline 0.2 & 1 & 1.50 & 2.06 & 2.31 & 2.42 \\
\hline 0.1 & 0.5 & 1.98 & 2.60 & 2.83 & 2.93 \\
\hline 0.02 & 0.1 & 3.34 & 4.08 & 4.27 & 4.36 \\
\hline 0.01 & 0.05 & 4.19 & 4.97 & 5.15 & 5.24 \\
\hline \hline
\end{tabular}

Table S2. Complete set of resolutions DoF for the single scattering albedo test, as panel (b) in Fig.4. 


\begin{tabular}{|c|c|c|c|c|c|c|}
\hline & & \multicolumn{5}{|c|}{ Aerosol scenario } \\
\hline \multicolumn{2}{|c|}{ Resolution } & Urban & Highly polluted & Elevated layer & Marine & Arctic \\
\hline$\Delta \nu$ & FWHM & $k_{e x t}=0.2 \mathrm{~km}^{-1}$ & $k_{e x t}=1 \mathrm{~km}^{-1}$ & $k_{e x t}=0.2 \mathrm{~km}^{-1}$ & $k_{e x t}=0.05 \mathrm{~km}^{-1}$ & $k_{e x t}=0.05 \mathrm{~km}^{-1}$ \\
\hline$\left[\mathrm{cm}^{-1}\right]$ & {$\left[\mathrm{cm}^{-1}\right]$} & $\mathrm{BLH}=1 \mathrm{~km}$ & $\mathrm{BLH}=1 \mathrm{~km}$ & Height $=0.6-1 \mathrm{~km}$ & $\mathrm{BLH}=0.6 \mathrm{~km}$ & $\mathrm{BLH}=0.6 \mathrm{~km}$ \\
\hline & & albedo $=0.1$ & albedo $=0.1$ & albedo $=0.1$ & albedo $=0.05$ & albedo $=0.9$ \\
\hline 1 & 5 & 2.01 & 2.11 & 1.40 & 1.19 & 0.34 \\
\hline 0.2 & 1 & 2.54 & 2.96 & 1.89 & 1.56 & 0.70 \\
\hline 0.1 & 0.5 & 3.03 & 3.37 & 2.33 & 1.92 & 1.32 \\
\hline 0.02 & 0.1 & 4.49 & 4.90 & 3.76 & 3.09 & 2.64 \\
\hline 0.01 & 0.05 & 5.38 & 5.92 & 4.60 & 3.84 & 3.43 \\
\hline
\end{tabular}

Table S3. Complete set of resolutions DoF for all the scenarios, as panel (a) in Fig.5. 


\begin{tabular}{ccccccc}
\hline \hline \multicolumn{1}{c}{} & \multicolumn{5}{c}{ Altitude range Urban scenario } \\
\hline \multicolumn{2}{c}{ Resolution } & & I & II & III & IV \\
\hline $\begin{array}{c}\Delta \nu \\
{\left[\mathrm{cm}^{-1}\right]}\end{array}$ & $\begin{array}{c}\text { FWHM } \\
{\left[\mathrm{cm}^{-1}\right]}\end{array}$ & Total DoF & $0-2[\mathrm{~km}]$ & $2-5[\mathrm{~km}]$ & $5-15[\mathrm{~km}]$ & $15-50[\mathrm{~km}]$ \\
\hline 1 & 5 & 2.01 & 0.36 & 0.65 & 0.20 & 0.80 \\
\hline 0.2 & 1 & 2.54 & 0.48 & 0.79 & 0.35 & 0.92 \\
\hline 0.1 & 0.5 & 3.03 & 0.59 & 1.01 & 0.45 & 0.98 \\
\hline 0.02 & 0.1 & 4.49 & 1.03 & 1.65 & 0.68 & 1.13 \\
\hline 0.01 & 0.05 & 5.38 & 1.48 & 1.96 & 0.75 & 1.19 \\
\hline \hline
\end{tabular}

Table S4. Complete set of resolutions DoF for the Urban scenario, as panel (b) in Fig.5. 


\begin{tabular}{ccccccc}
\hline \hline \multicolumn{2}{c}{} & \multicolumn{5}{c}{ Altitude range Highly polluted scenario } \\
\hline \multicolumn{2}{c}{ Resolution } & \multicolumn{1}{c}{ I } & II & III & IV \\
\hline $\begin{array}{c}\Delta \nu \\
{\left[\mathrm{cm}^{-1}\right]}\end{array}$ & $\begin{array}{c}\text { FWHM } \\
{\left[\mathrm{cm}^{-1}\right]}\end{array}$ & Total DoF & $0-2[\mathrm{~km}]$ & $2-5[\mathrm{~km}]$ & $5-15[\mathrm{~km}]$ & $15-50[\mathrm{~km}]$ \\
\hline 1 & 5 & 2.11 & 0.41 & 0.79 & 0.28 & 0.62 \\
\hline 0.2 & 1 & 2.96 & 0.65 & 1.08 & 0.43 & 0.81 \\
\hline 0.1 & 0.5 & 3.37 & 0.73 & 1.27 & 0.49 & 0.88 \\
\hline 0.02 & 0.1 & 4.90 & 1.16 & 1.98 & 0.74 & 1.02 \\
\hline 0.01 & 0.05 & 5.92 & 1.70 & 2.34 & 0.81 & 1.07 \\
\hline \hline
\end{tabular}

Table S5. Complete set of resolutions DoF for the Highly polluted scenario, as panel (c) in Fig.5. 


\begin{tabular}{ccccccc}
\hline \hline & \multicolumn{5}{c}{ Altitude range Elevated layer scenario } \\
\hline \multicolumn{2}{c}{ Resolution } & \multicolumn{1}{c}{ I } & II & III & IV \\
\hline $\begin{array}{c}\Delta \nu \\
{\left[\mathrm{cm}^{-1}\right]}\end{array}$ & $\begin{array}{c}\text { FWHM } \\
{\left[\mathrm{cm}^{-1}\right]}\end{array}$ & Total DoF & $0-2[\mathrm{~km}]$ & $2-5[\mathrm{~km}]$ & $5-15[\mathrm{~km}]$ & $15-50[\mathrm{~km}]$ \\
\hline 1 & 5 & 1.40 & 0.15 & 0.26 & 0.05 & 0.93 \\
\hline 0.2 & 1 & 1.89 & 0.31 & 0.43 & 0.06 & 1.09 \\
\hline 0.1 & 0.5 & 2.33 & 0.46 & 0.55 & 0.08 & 1.24 \\
\hline 0.02 & 0.1 & 3.76 & 0.87 & 1.17 & 0.18 & 1.53 \\
\hline 0.01 & 0.05 & 4.60 & 1.27 & 1.46 & 0.22 & 1.65 \\
\hline \hline
\end{tabular}

Table S6. Complete set of resolutions DoF for the Elevated layer scenario, as panel (d) in Fig.5. 


\begin{tabular}{ccccccc}
\hline \hline \multicolumn{2}{c}{} & \multicolumn{5}{c}{ Altitude range Marine scenario } \\
\hline \multicolumn{2}{c}{ Resolution } & \multicolumn{1}{c}{ I } & II & III & IV \\
\hline $\begin{array}{c}\Delta \nu \\
{\left[\mathrm{cm}^{-1}\right]}\end{array}$ & $\begin{array}{c}\text { FWHM } \\
{\left[\mathrm{cm}^{-1}\right]}\end{array}$ & Total DoF & $0-2[\mathrm{~km}]$ & $2-5[\mathrm{~km}]$ & $5-15[\mathrm{~km}]$ & $15-50[\mathrm{~km}]$ \\
\hline 1 & 5 & 1.19 & 0.24 & 0.44 & 0.08 & 0.42 \\
\hline 0.2 & 1 & 1.56 & 0.33 & 0.52 & 0.10 & 0.61 \\
\hline 0.1 & 0.5 & 1.92 & 0.43 & 0.62 & 0.17 & 0.70 \\
\hline 0.02 & 0.1 & 3.09 & 0.80 & 1.10 & 0.34 & 0.86 \\
\hline 0.01 & 0.05 & 3.84 & 1.18 & 1.36 & 0.40 & 0.91 \\
\hline \hline
\end{tabular}

Table S7. Complete set of resolutions DoF for the Marine scenario, as panel (e) in Fig.5. 


\begin{tabular}{|c|c|c|c|c|c|c|}
\hline & & & \multicolumn{4}{|c|}{ Altitude range Arctic scenario } \\
\hline \multicolumn{2}{|c|}{ Resolution } & & I & II & III & IV \\
\hline $\begin{array}{c}\Delta \nu \\
{\left[\mathrm{cm}^{-1}\right]}\end{array}$ & $\begin{array}{l}\text { FWHM } \\
{\left[\mathrm{cm}^{-1}\right]}\end{array}$ & Total DoF & $0-2[\mathrm{~km}]$ & $2-5[\mathrm{~km}]$ & $5-15[\mathrm{~km}]$ & $15-50[\mathrm{~km}]$ \\
\hline 1 & 5 & 0.34 & 0.02 & 0.05 & 0.04 & 0.23 \\
\hline 0.2 & 1 & 0.70 & 0.09 & 0.15 & 0.07 & 0.38 \\
\hline 0.1 & 0.5 & 1.32 & 0.36 & 0.43 & 0.08 & 0.46 \\
\hline 0.02 & 0.1 & 2.64 & 0.80 & 1.02 & 0.19 & 0.64 \\
\hline 0.01 & 0.05 & 3.43 & 1.18 & 1.31 & 0.23 & 0.71 \\
\hline
\end{tabular}

Table S8. Complete set of resolutions DoF for the Arctic scenario, as panel (f) in Fig.5. 


\begin{tabular}{ccccccc}
\hline \hline & \multicolumn{6}{c}{$\boldsymbol{S}_{a}$ (relative error) } \\
\hline Integration time & 0.7 & 0.5 & 0.2 & 0.1 & 0.05 & 0.01 \\
\hline$\Delta t[\mathrm{sec}]$ & $(83.7 \%)$ & $(70.7 \%)$ & $(44.7 \%)$ & $(31.6 \%)$ & $(22.4 \%)$ & $(10.0 \%)$ \\
\hline 0.1 & 4.55 & 4.36 & 3.83 & 3.42 & 3.01 & 2.03 \\
\hline 0.2 & 4.75 & 4.56 & 4.03 & 3.63 & 3.22 & 2.24 \\
\hline 0.5 & 5.03 & 4.83 & 4.29 & 3.89 & 3.49 & 2.53 \\
\hline 1 & 5.24 & 5.03 & 4.49 & 4.09 & 3.69 & 2.74 \\
\hline 2 & 5.46 & 5.25 & 4.69 & 4.29 & 3.89 & 2.95 \\
\hline 5 & 5.78 & 5.54 & 4.97 & 4.56 & 4.16 & 3.22 \\
\hline \hline
\end{tabular}

Table S9. DoF for integration time sensitivity for Urban scenario for different $\boldsymbol{S}_{a}$, as panel (a) in Fig.6. 


\begin{tabular}{cccccc}
\hline \hline & \multicolumn{5}{c}{ Urban scenario } \\
\hline Integration time & \multicolumn{5}{c}{ FWHM $\left[\mathrm{cm}^{-1}\right]$} \\
\hline$\Delta t[\mathrm{sec}]$ & 5 & 1 & 0.5 & 0.1 & 0.05 \\
\hline 0.1 & 1.73 & 2.12 & 2.60 & 3.83 & 4.59 \\
\hline 0.2 & 1.82 & 2.24 & 2.73 & 4.03 & 4.83 \\
\hline 0.5 & 1.93 & 2.40 & 2.90 & 4.29 & 5.14 \\
\hline 1 & 2.01 & 2.54 & 3.03 & 4.49 & 5.38 \\
\hline 2 & 2.09 & 2.68 & 3.16 & 4.69 & 5.63 \\
\hline 5 & 2.19 & 2.88 & 3.35 & 4.97 & 5.97 \\
\hline
\end{tabular}

Table S10. Complete set of resolutions DoF for integration time sensitivity for Urban scenario for different resolution, as panel (b) in Fig.6. 